\title{
Colonoscopia em Pacientes não Pediátricos Abaixo de 20 Anos de Idade traz pouca Contribuição nos Resultados
}

\author{
Colonoscopy in Non Pediatric Patients Under 20 Years Old Brings Poor \\ Contribution in Results
}

\author{
ANTONIO SÉRGIO BRENNER ${ }^{1}$; VANESSA ZENI LIMA²; SANDRABEATRIZ MARION VALARINI; \\ RUBENS VALARINI ${ }^{4}$;ANA MARIA PEREIRACÉSAR ${ }^{5}$
}

\begin{abstract}
${ }^{1}$ Professor Adjunto e Médico do Serviço de Coloproctologia do Hospital Universitário Evangélico de Curitiba; ${ }^{2}$ Residente do Serviço de Coloproctologia do Hospital Universitário Evangélico de Curitiba; ${ }^{3}$ Professora Assistente da Pontifícia Universidade Católica do Paraná; ${ }^{4}$ Médico do Serviço de Coloproctologia do Hospital Universitário Evangélico de Curitiba; ${ }^{5}$ Médico do Instituto do Aparelho Digestivo de Curitiba.
\end{abstract}

\begin{abstract}
BRENNER AS; LIMA VZ; VALARINI SBM;; VALARINI R; CÉSAR AMP. Colonoscopia em Pacientes Não Pediátricos Abaixo de 20 Anos de Idade Traz Pouca Contribuição nos Resultados. Rev bras Coloproct, 2007;27(4): 412-416.

RESUMO: Introdução: A utilização da colonoscopia como método de diagnóstico e prevenção das doenças do cólon e reto é cada ano mais freqüente. Esse aumento na indicação também é observado nos extremos de idade, como pacientes não pediátricos abaixo dos 20 anos. Método: Entre outubro de 1998 a julho de 2006, 4354 pacientes foram submetidos à colonoscopia na clínica privada de um dos autores. Foram analisados retrospectivamente, os prontuários de 66 pacientes com vinte anos ou menos de idade. $O$ estudo histológico foi obtido nos pacientes com exames alterados. Resultados: A idade variou de 11 a 20 anos (média de 17,96 anos). Trinta e nove pacientes pertenciam ao sexo feminino e 27 ao masculino. $O$ exame foi normal em 40 , observou-se apenas hiperplasia de tecido linfóide em 10, divertículos em 2, colite inespecífica em 1 e pólipo hiperplásico em 1 paciente, totalizando 54 (81,81\%) exames sem alteração específica. Colites foram observadas em 10 e pólipos em 5 . Em dois pacientes havia história familiar de doença inflamatória intestinal e em um de câncer de cólon. Conclusão: A seleção dos pacientes é fundamental para evitar exames colonoscópicos desnecessários em pacientes abaixo de 20 anos de idade.
\end{abstract}

Descritores: Colonoscopia; adolescente; rastreamento; colo; diagnóstico.

\section{INTRODUÇÃO}

A colonoscopia é um método diagnóstico cada vez mais utilizado na avaliação e prevenção das doenças do cólon e reto. A importância da avaliação colorretal tornou-se tão importante que é hoje utilizada em programas de rastreamento do câncer do intestino na população geral e assintomática. É um exame que pode além de diagnosticar e tratar, também prevenir o câncer colorretal através da ressecção de pólipos ${ }^{1-4}$. A possibilidade de prevenção é o que difere o rastreamento do câncer colorretal do exame da mama ou próstata, onde o objetivo final é meramente o diagnóstico precoce da neoplasia já instalada.

Ainda não existe método diagnóstico comparável com colonoscopia endoscópica. A colonoscopia virtual, mesmo melhorando sensivelmente seus resultados, será eternamente dependente da endoscópica para confirmação diagnóstica ou tratamento definitivo das lesões. O enema opaco tornou-se hoje um exame utilizado em casos selecionados de doença diverticular, situações em que é necessário uma avaliação anatômica da conformação colônica ou casos de impossibilidade de realização da colonoscopia até o ceco. O

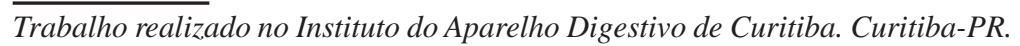

Recebido em 18/10/2007

Aceito para publicação em 13/11/2007 
rastreamento do câncer ou pólipos através do enema caiu em desuso pela elevada incidência de falsos negativo e positivo. Uma série compara a colonoscopia com o enema opaco. Quinhentos e oitenta pacientes foram avaliados em 862 exames. A colonoscopia foi, em média, realizada 16 dias após o enema opaco. O exame endoscópico detectou mais pólipos. Em torno de $20 \%$ dos adenomas não foram identificados no enema opaco 5 .

O exame endoscópico pode ser realizado em qualquer faixa etária. Mesmo nos extremos de idade - como os pacientes acima de 80 ou abaixo dos 20 anos. Algumas publicações avaliaram os resultados dos exames realizados em pacientes idosos. Na grande maioria, esses estudos verificaram as necessidades e os resultados no rastreamento endoscópico do câncer ou então avaliaram os riscos inerentes ao procedimento $^{6-8}$.

A finalidade do atual estudo foi questionar a indicação dos exames colonoscópicos, baseando-se nos resultados obtidos nos adolescentes. Pacientes não pediátricos (acima de 15 anos) abaixo de 20 anos de idade. Nessa faixa etária está um grupo de pacientes em que, pela raridade na incidência de doenças, o exame da colonoscopia, traria essencialmente um aumento no custo e exposição dos pacientes aos riscos do procedimento.

\section{MÉTODO}

Avaliamos retrospectivamente os prontuários de 4354 pacientes submetidos à colonoscopia na clínica privada de um dos autores, realizados entre outubro de 1998 a julho de 2006.

Sessenta e seis pacientes possuíam entre onze e vinte anos de idade, e esse foi o grupo de pacientes objeto do presente estudo. Os dados avaliados foram: idade, sexo, resultado do exame e do estudo histológico. A indicação do exame, história pregressa e familiar do paciente não foi obtida em 46 pacientes em que não foi possível o acesso ao prontuário.

Todos os exames foram realizados por um dos autores, sob sedação profunda utilizando-se propofol endovenoso sob infusão direta com assistência de anestesista. Todos os examinadores possuem extensa experiência em colonoscopia, cada um com mais de 1000 exames realizados. Foram utilizados colonoscópios padrão de $160 \mathrm{~cm}$ das marcas Olympus, Pentax ou Fujiinon.
Os pacientes receberam preparo de cólon por dieta restrita, ingestão de bisacodil $10 \mathrm{mg}$, solução de manitol a 10\% num volume de $1000 \mathrm{ml}$ e fleet enema via retal no dia anterior ao exame. Os exames com preparo intestinal inadequado foram cancelados e excluídos do estudo ou novamente agendados e repetidos em melhores condições de preparo.

Foram obtidas biópsias dos pacientes com exames alterados, e o estudo histológico realizado por um de dois patologistas, sob técnica histológica padrão da hematoxilina-eozina. Em 6 pacientes, não obtivemos o resultado histopatológico (dois pacientes com pólipo e 4 com colite).

\section{RESULTADOS}

Em todos os 66 casos, a colonoscopia foi completa com intubação cecal.

Não houveram complicações anestésicas ou relacionadas ao procedimento.

Dos 66 pacientes avaliados, trinta e nove (59\%) pacientes pertenciam ao sexo feminino e 27 (41\%) ao masculino. A idade média foi de 17,96 anos (variando de 11 a 20 anos).

A indicação do procedimento foi obtida em 20 pacientes, sendo: a enterorragia em 9 casos e a suspeita de doença inflamatória intestinal em 4 , a síndrome do intestino irritável em 2 e distensão abdominal em 2 pacientes. Em dois pacientes havia história familiar de doença inflamatória intestinal e em um de câncer de cólon. Gráfico 1

Analisando-se os resultados, o exame foi normal em 40 pacientes. Observou-se apenas hiperplasia de tecido linfóide no íleo terminal em 10, divertículos não complicados em 2, colite inespecífica em 1 e pólipo hiperplásico em 1 paciente. Totalizando portanto, 54 pacientes sem alteração específica. Em doze casos, foi observada alguma alteração endoscópica com possível repercussão clínica: colites específicas em 8, pólipos não hiperplásicos em 4. Gráfico 2.

Foi observado um caso de colite eosinofílica num paciente com colite. Um caso de displasia de baixo grau foi identificado em paciente com RCUI. Colite por cândida ocorreu em um caso de diarréia crônica. Um caso de colite por Crohn e um de colite inespecífica. Entre as polipectomias evidenciou-se um pólipo hiperplásico, um pólipo juvenil e um adenoma tubular. O estudo histopatológico não foi obtido em 4 pacientes com colite. Em dois pacientes com pólipo 
não foi possível a recuperação do espécime para estudo histológico. Tabela 1.

\section{DISCUSSÃO}

Do total de 66 pacientes, 54 apresentaram exames sem resultado positivo específico - exames normais, com hiperplasia linfóide e divertículos. Dos 12 pacientes com alteração no exame (não conseguimos uma avaliação histopatológica em 6 casos), apenas seis apresentaram uma histologia alterada - displasia, colites e pólipos. Portanto, apenas $9,09 \%$ dos exames indicados em pacientes abaixo de 20 anos apresentaram alguma alteração justificando o exame. Supondo que os restantes 6 pacientes (em que não obtivemos à avalia-
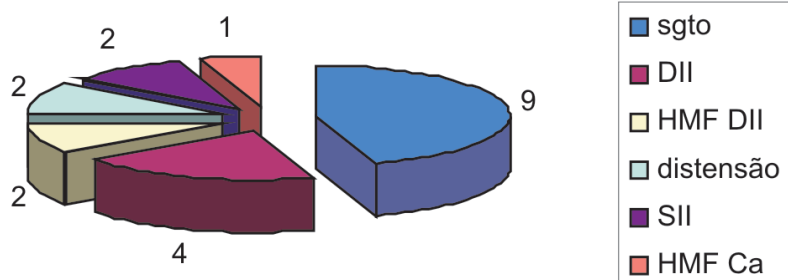

Gráfico 1 - Indicação do exame.

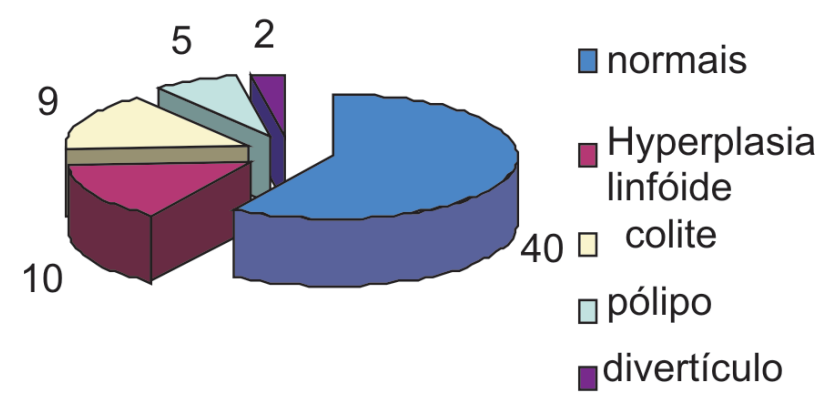

Gráfico 2 - Resultado do exame.

Tabela 1.

\begin{tabular}{lrcc}
\hline & N & $\begin{array}{c}\text { Histologia } \\
\text { Alterada }\end{array}$ & $\%$ \\
\hline Exames normais & 40 & - & $52(78,78 \%)$ \\
Hiperplasia Linfóide & 10 & - & \\
Divertículos & 2 & - & \\
Colites & 9 & 4 & $14(21,21 \%)$ \\
Pólipos & 5 & 2 & \\
\hline
\end{tabular}

ção histológica), apresentassem exames com alteração específica (excluindo-se colites inespecíficas e pólipos hiperplásicos por exemplo), mesmo assim, apenas $18,18 \%$ dos exames colonoscópicos trariam um benefício ao tratamento. Analisado nossos resultados, observamos que em poucos casos o exame confirmou um diagnóstico histológico específico, que fosse determinante no tratamento ou mudança de conduta clínica.

A literatura mundial é pobre em publicações, avaliando os achados da colonosocopia em pacientes adolescentes ou mesmo em crianças. Os poucos autores que direcionaram sua curiosidade à essa população, preferiram avaliar doenças específicas como síndromes polipóides, necessidade de rastreamento do câncer hereditário e relatos de casos raros ou patologias exóticas ${ }^{1,9-15}$. No outro extremo de idade, o de pacientes idosos acima de 60 anos, o número de publicações aumenta vertiginosamente. Esse fato é compreensível pela maior incidência de patologias, especialmente o câncer, nessa faixa etária ${ }^{6-8,12}$. Por outro lado, deixa a questão da indicação da colonoscopia em jovens ainda não respondidas.

Percebemos a escassez de publicações revisando os poucos estudos em colonoscopia de pacientes jovens. Avaliamos os nossos resultados através dos exames realizados em clínica particular que atende, estritamente, pacientes conveniados ao sistema privado de saúde ou particulares pagantes, que foram encaminhados por colegas ou atendidos na própria instituição. Essa população não representa uma avaliação fiel da população geral. Aproximadamente $75 \%$ dos pacientes brasileiros buscam atendimento no sistema público de saúde ${ }^{16}$. Como nossos pacientes têm uma condição sócio-econômica mais alta, seria esperado um maior número de exames normais, doenças em estágio mais inicial ou até diferenças no perfil epidemiológico. Maior incidência de doença inflamatória intestinal e raros casos de megacólon.

Nossos resultados mostram que ao menos $81,81 \%$ dos pacientes têm exame normal (inclui pacientes com exame normal, hiperplasia de tecido linfóide no íleo terminal, divertículos, colite inespecífica e pólipo colorretal hiperplásico). Um grande contraste com a incidência de achados anormais na população geral ${ }^{6-8}$, ${ }^{17}$. Uma revisão realizada por Bowles e colaboradores, publicada em 2004, que demonstrou incidência de $42,1 \%$ de exames normais, pólipos em $22 \%$, doença inflama- 
tória intestinal em $13,9 \%$ e câncer em $3,8 \%$. O estudo abrangeu uma população geral de 9.223 pacientes sendo: pediátricos $(<16$ anos $)=1,8 \%$; adultos $=81,5 \%$; idosos $(>75$ anos $)=14,1 \%$ e de idade desconhecida em $2,6 \%{ }^{18}$.

Adolescentes situam-se numa faixa etária desprovida de atendimento especializado. Ficam isolados entre o atendimento do cirurgião pediátrico e o do coloproctologista ou entre o pediatra e o gastroenterologista. A indicação adequada da colonoscopia em jovens permanece sem definição e conseqüentemente, algumas perguntas sem resposta adequada: Deveríamos indicar a colonoscopia em todos os casos de enterorragia ou somente naqueles com sangramento sem causa orificial definida? Qual o momento de indicar a colonoscopia nos casos de alteração do hábito intestinal (diarréia) nos jovens? Existe indicação no rastreamento das famílias com doença inflamatória intestinal? São inúmeras as questões ainda não bem esclarecidas.

Nossa expectativa é iniciar uma discussão. Estimular futuros levantamentos sobre as indicações e os resultados da colonoscopia em adolescentes onde, represente um benefício mais evidente, dentro do cenário de custos penosamente toleráveis da medicina moderna.

\section{CONCLUSÃO}

A colonoscopia em pacientes abaixo de 20 anos de idade apresenta baixo índice de resultados importantes e decisivos na conduta clínica. A seleção dos pacientes é fundamental para evitar exames desnecessários.

\footnotetext{
ABSTRACT: Introduction: The colonoscopy as a diagnostic and prevention tool has been increasingly used for the prevention and treatment of the colon and rectum diseases. This rise is also seen in non pediatric teenagers under 20 years old. Method: Between October 1998 and July 2006, 4354 patients were submitted to colonoscopy scope in one of the author's privet clinic. The files of 66 patients under 20 y/o were retrospectively analyzed. Histology was obtained for patients with abnormal exams. Results: Medium age was 17,96 y/o (range 11 to 20). Thirty nine patients were female and 27 male. The exam was normal in 40, Linfoid hiperplasic tissue was found in 10, diverticulosis in 2, unspecific colitis in 1 and hyperplasic polyps in 1 patient, totalizing 54 $(\mathbf{8 1 , 8 1 \% )}$ ) exams without any specific alteration. Colitis was observed in 10 and polyps in 5 . Two patients have family history of IBD and one of colon cancer. Conclusion: Patient selection is crucial to avoiding unnecessary exams in non-pediatric patients under 20 years old.
}

Key words: Colonoscopy; adolescent; screening; colon; diagnosis.

\section{REFERÊNCIAS}

1. Gilbert JM, Vaizey CJ, Cassell PG, Holden J. Feasibility study of colonoscopy as the primary screening investigation in relatives of patients with colorectal cancer. Ann R Coll Surg Engl 2001;83(6):415-9.

2. McCloy R, Nagengast F, Fried M, Rohde H, Froehlich F, Whitwam J. Conscious sedation for endoscopy. Eur J Gastroenterol Hepatol 1996;8(12):1233-40.

3. Nagengast FM. Sedation and monitoring in gastrointestinal endoscopy. Scand J Gastroenterol Suppl 1993;200:28-32.

4. Thomas-Gibson S, Thapar C, Shah SG, Saunders BP. Colonoscopy at a combined district general hospital and specialist endoscopy unit: lessons from 505 consecutive examinations. J R Soc Med 2002;95(4):194-7.

5. Winawer SJ, Stewart ET, Zauber AG, et al. A comparison of colonoscopy and double-contrast barium enema for surveillance after polypectomy. National Polyp Study Work Group. N Engl J Med 2000;342(24):1766-72.

6. Cooper GS. Con: screening colonoscopy in the extreme elderly is not a wise choice. Am J Gastroenterol 2006;101(8):1715-7; discussion 7-8.

7. Kung JW, Levine MS, Glick SN, Lakhani P, Rubesin SE, Laufer I. Colorectal cancer: screening double-contrast barium enema examination in average-risk adults older than 50 years. Radiology 2006;240(3):725-35.

8. Schoen RE. Pro: should screening colonoscopy be performed on an 88-yr-old healthy patient? Am J Gastroenterol 2006;101(8):1713-5; discussion 7-8.

9. Arain Z, Rossi TM. Gastrointestinal bleeding in children: an overview of conditions requiring nonoperative management. Semin Pediatr Surg 1999;8(4):172-80.

10. Chikhaoui Y, Gelinas H, Joseph L, Lance JM. Costminimization analysis of genetic testing versus clinical screening 
of at-risk relatives for familial adenomatous polyposis. Int $\mathbf{J}$ Technol Assess Health Care 2002;18(1):67-80.

11. Dean PA. Hereditary intestinal polyposis syndromes. Rev Gastroenterol Mex 1996;61(2):100-11.

12. Eisinger F, Giordanella JP, Brigand A, et al. Cancer prone persons. A randomized screening trial based on colonoscopy: background, design and recruitment. Fam Cancer 2001;1(34):175-9.

13. Markowitz J, McKinley M, Kahn E, et al. Endoscopic screening for dysplasia and mucosal aneuploidy in adolescents and young adults with childhood onset colitis. Am J Gastroenterol 1997;92(11):2001-6.

14. Ofiaeli RO. Volvulus of the sigmoid colon in paediatric patients: report of two cases. Cent Afr J Med 1992;38(4):169-71.

15. Yamamoto K, Tanaka T, Kuno K, Amoh Y, Takahashi Y, Murakami H. Carcinoma of the colon in children: case report and review of the Japanese literature. J Gastroenterol 1994;29(5):647-52.
16. IBGE IBdGeE-. 2006.

17. Shih YC, Zhao L, Elting LS. Does Medicare coverage of colonoscopy reduce racial/ethnic disparities in cancer screening among the elderly? Health Aff (Millwood) 2006;25(4):1153-62.

18. Bowles CJ, Leicester R, Romaya C, Swarbrick E, Williams $\mathrm{CB}$, Epstein O. A prospective study of colonoscopy practice in the UK today: are we adequately prepared for national colorectal cancer screening tomorrow? Gut 2004;53(2):277-83.

\section{Endereço para correspondência:}

DR. ANTONIO SÉRGIO BRENNER

Rua de Paz, 156

Curitiba - PR - Brasil

80060160

Tel / FAX: 554132634474

E-mail:medicos@iadcuritiba.com.br 